\title{
Philosophiques
}

\section{Sven Walter et Heinz-Dieter Heckmann (dir.), Physicalism and mental causation: The Metaphysics of Mind and Action, Exeter et Charlottesville, Imprint Academic, 2003, 362 pages.}

\section{François Loth}

Volume 32, numéro 2, automne 2005

URI : https://id.erudit.org/iderudit/011887ar

DOI : https://doi.org/10.7202/011887ar

Aller au sommaire du numéro

Éditeur(s)

Société de philosophie du Québec

ISSN

0316-2923 (imprimé)

1492-1391 (numérique)

Découvrir la revue

Citer ce compte rendu

Loth, F. (2005). Compte rendu de [Sven Walter et Heinz-Dieter Heckmann (dir.), Physicalism and mental causation: The Metaphysics of Mind and Action, Exeter et Charlottesville, Imprint Academic, 2003, 362 pages.] Philosophiques, 32(2),

479-483. https://doi.org/10.7202/011887ar d'utilisation que vous pouvez consulter en ligne.

https://apropos.erudit.org/fr/usagers/politique-dutilisation/ 
l'extension" d'une représentation. L'extension de la représentation désignée par l'expression «Socrate» n'est pour Bolzano rien d'autre que l'objet de cette représentation. Corrélativement, que l'on puisse, en un sens particulier, attribuer une extension à une représentation sans objet signifie eo ipso qu'on lui attribue un objet. Il n'y a pas, chez Bolzano, de distinction d'essence entre extension et objet, comme l'affirme l'auteur.

Toujours au second chapitre (p. 264), l'auteur prétend qu'en critiquant la théorie des assomptions de Meinong dans les Idées, I, Husserl en vient par le fait même à critiquer la position sur les assomptions telle qu'elle était exposée dans les $R l$. Pour cela, il s'appuie sur une note de bas de page des Idées, I, qui rectifie le statut de qualité d'acte de la neutralité pour en faire une modification d'acte. Or cette note de bas de page dit trop peu pour pouvoir appuyer la thèse de Seron. Au contraire, Husserl se distancie de la théorie des assomptions de Meinong dès la lecture de Über Annahmen en 1902, soit un an après la publication des Recherches logiques, dans une lettre adressée à Meinong (voir Husserl, Briefwechsel, vol. 2: die Brentano-Schule, p. 142-144). À la même lettre, il adjoignit alors le manuscrit de Objets intentionnels de 1894, afin de montrer à Meinong qu'il avait développé une théorie similaire de la supposition longtemps avant celle de Über Annahmen. En ce sens, et contre l'auteur, la critique husserlienne de la théorie meinongienne des assomptions n'est pas à considérer comme allant de pair avec un dépassement des $R l$, dans la mesure où son origine est à trouver déjà dans des écrits antérieurs à la publication des Recherches.

Dans l'ensemble, il est juste de dire que Seron propose une étude approfondie de la question du jugement dans les développements de la phénoménologie allant des $R l$ à Expérience et jugement. L'ouvrage aborde de manière non linéaire les différentes étapes de la théorie phénoménologique du jugement en se concentrant en grande partie sur les ouvrages publiés du vivant de Husserl. Cette stratégie a l'avantage de permettre à l'auteur de former son propos plus librement que ne l'aurait permis une étude exégétique, mais elle laisse de côté des textes qui auraient été significatifs dans le cadre de cette étude, notamment une quantité importante de manuscrits de Husserl consacrés à la question, ainsi que les Leçons de 1905 sur la théorie du jugement (Husserl, Urteilstheorie, 1905; Dordrecht, 2002). Malgré ce déficit, l'ouvrage contient des informations précieuses qui pourront aider à la compréhension des enjeux de la théorie du jugement en phénoménologie.

GUILLAUME FRÉCHETTE Université de Hambourg

\section{Sven Walter et Heinz-Dieter Heckmann (dir.), Physicalism and mental causation: The Metaphysics of Mind and Action, Exeter et Charlottesville, Imprint Academic, 2003, 362 pages.}

Les quatorze textes rassemblés dans l'ouvrage de Sven Walter et Heinz-Dieter Heckmann traitent tout un ensemble de notions spécifiques au domaine de la philosophie de l'esprit (la réalisation multiple, la survenance, la surdétermination et la clôture causale du domaine physique, l'exclusion causale explicative, etc.) impliquant la causation mentale. Cette unité dans la discussion que nous propose chaque texte 
est apportée par le lien problématique et séculaire de la relation du domaine mental au domaine physique.

Le problème de la causation mentale apparaît dès que l'on tente de répondre à la question de la possibilité pour un esprit d'effectuer un changement dans le monde physique. À travers quel mécanisme puis-je rendre compte de la connexion entre mon désir d'écrire cette phrase et le mouvement de mes doigts sur le clavier de l'ordinateur?

En abordant ces questions, la philosophie de l'esprit s'intéresse à la fois à la nature des propriétés mentales et au lien que de telles propriétés peuvent entretenir avec notre monde physique.

Bien que le vingtième siècle ait connu une certaine hégémonie du physicalisme - affirmation que chaque chose se trouvant dans le monde est physique, y compris les esprits humains - il n'en demeure pas moins que dans le contexte physicaliste persiste un certain nombre d'obstacles qui permettent de rendre compte de nos activités mentales. Parmi les problèmes et obstacles que l'on peut recenser se pose le problème du lien entre les propriétés dont chacune appartient à un monde particulier. Dès que l'on a contesté leur identité avec les propriétés physiques, les propriétés mentales sont-elles réalisées, déterminées ou constituées par les propriétés physiques? La première partie du livre réunit cinq textes discutant cet aspect conceptuel du lien entre les deux types de propriétés: Multiply Realized Properties, de John Heil; NonReductive Realization and Non-Reductive Identity: What Physicalism Does Not Entail, de Carl Gillet; Multiple Realizability an Psychological Laws : Evaluating Kim's Challenge, de Gene Witmer; Not Old... But Not That Either: Explicability, Emergence, and the Characterisation of Materialism, de Paul Noordhof; A Farewell to Isms, de John Bolender. Les trois autres parties de l'ouvrage collectif, quant à elles, (Overdetermination and the Causal Closure of the Physical-Mental Causation and the Problem of Causal/Explanatory Exclusion - Causality and Human Agency) soulèvent une série de questions relatives à la causation mentale. Il s'agit d'un côté de débattre de l'écart que l'expérience quotidienne produit entre nos actions d'agents autonomes, mues par ce que nous nommons croyances et désirs, et de l'autre, de donner, si le physicalisme est vrai, une description physique suffisante de chacune de nos actions. Comment accorder ces deux images ensemble? Comment peuvent-elles être simplement des descriptions d'une et même réalité?

Notre compte rendu se focalise sur la première partie intitulée Fondations conceptuelles: réalisation, survenance et caractérisation du physicalisme.

Lorsque J. J. C. Smart et U. T. Place avancèrent la théorie de l'identité espritcerveau et proclamèrent que le mental n'est rien de plus que le physique, ils établirent que les propriétés mentales et les propriétés physiques (neuronales) étaient identiques. Il semblait cependant fort peu vraisemblable que les propriétés mentales, comme ressentir de la douleur ou croire que Lille est situé au nord de Paris, puissent être identiques à des propriétés physiques. En effet, un argument introduit par H. Putnam, celui de la réalisation multiple, allait rapidement mettre à mal la théorie de l'identité psychophysique. Il paraît effectivement crédible que les propriétés mentales se réalisent différemment sur le plan physique, selon les individus ou les espèces, ou dans le même individu à divers moments.

La résistance de l'esprit réductionniste, que l'argument de la réalisation multiple attaquait, allait trouver une sorte de solution dans l'introduction de la notion de propriétés disjonctives. Étant donné que les propriétés mentales se réalisent de mul- 
tiples façons, une réduction du mental pouvait malgré tout s'opérer et prendre la forme d'une série de différentes propriétés disjonctives $\left(P_{1}, \ldots P_{n}\right)$. Selon le modèle classique de la réduction de E. Nagel, nous obtiendrions une loi-pont (LP) entre les propriétés à réduire $(M)$ et les propriétés réduites $(P)$ comme celle-ci:

$$
\text { (LP) }(\forall x)\left(M x_{-} P_{1} x \vee \ldots \vee P_{n} x\right)
$$

J. Fodor et H. Putnam objectèrent à ce modèle réductionniste qu'un groupe aussi hétérogène de propriétés ne pouvait désigner un véritable genre naturel. En conséquence, comme on ne pouvait identifier les propriétés mentales $(M)$ à leurs réalisateurs $(P)$, un physicalisme non réductif fut alors revendiqué. C'est ainsi que la question du lien entre ces propriétés mentales irréductibles et le principe de leur réalisation allait se voir justifier par l'introduction d'un certain nombre de notions, telles que la survenance, la dépendance ou encore, la réalisation.

Ce sera le philosophe J. Kim, qui, partant des conclusions des thèses non réductives, développera dans un certain nombre de travaux une mise en cause de la pertinence du caractère irréductible des propriétés. En effet, sous l'égide d'une métaphysique matérialiste conséquente, Kim affirmera que le véritable travail causal est effectué par les propriétés du principe réalisateur. Après qu'il eut ajouté, dans cette thèse, qu'attribuer un critère de réalité aux seules propriétés dotées d'un pouvoir causal constitue un lien cohérent, il n'en fallait pas plus pour faire entrer Kim dans le camp des éliminativistes. Mais l'objectif de Kim (Mind in a Physical World: An Essay on The Mind-Body Problem and Mental Causation), guetté à la fois par un épiphénoménisme à fort relent sceptique et un éliminativisme radical, était de garder le mental à l'intérieur du monde physique.

Les trois premiers articles de ce livre portent sur les travaux de J. Kim.

L'argument principal de Kim consiste à affirmer que la réalisation est à la racine de la réduction et que, par conséquent, la notion même de "propriétés de niveau supérieur, irréductibles», n'a pas de sens véritable. Gene Witmer, dans son article intitulé "Multiple Realizability and Psychological Laws», estime que les raisons invoquées par Kim pour fonder son argument ne permettent pas de conclure que la multiplicité de réalisations du principe sous-jacent au mental forme un obstacle à l'établissement de lois psychologiques. La disjonction des propriétés physiques sousjacentes qui les empêche de figurer dans les lois entraînerait, selon Kim, l'impossibilité pour les prédicats $\mathrm{M}$ de véritablement se projeter. Witmer considère alors que l'on doit dissocier le caractère projectif d'un prédicat des propriétés classées comme disjonctives.

Witmer souligne le lien entre les lois de niveau supérieur et les lois des différents réalisateurs sous-jacents. Pour Kim, les lois qui gouvernent $M$ doivent tirer leur explication, c'est-à-dire leur pouvoir causal, des lois issues du principe de réalisation $P$. Mais à partir du moment où la propriété de niveau supérieur est réalisée par une diversité de propriétés physiques, comment une telle dérivation est-elle possible? Selon Witmer, bien que les propriétés fonctionnelles ne soient pas des propriétés basiques, les lois qui gouvernent ces propriétés de niveau supérieur doivent dépendre de lois régissant les propriétés plus basiques. Le genre d'explication recherché est donc la dérivation. Outre le fait de savoir si nous pouvons produire une telle explication, notre confiance dans les lois régissant les propriétés fonctionnelles dépend de notre confiance dans cette dérivation. Mais devrions-nous être si confiant, demande Witmer? Une telle explication de la dérivation entre les propriétés de niveau basique et les 
propriétés de niveau supérieur est pour l'auteur, quasi improbable. Comment une simple loi, mettant en jeu la propriété mentale de la douleur, pourrait-elle être dérivée de cette grande variété de lois qui gouvernent les différents réalisateurs physiques?

Dans la troisième partie, Witmer évoque l'hypothèse d'une projection de cas basée sur la réalisation multiple. Une telle orientation nous engagerait vers une prolifération d'explications indépendantes les unes des autres. Le philosophe établit alors, dans son «argument de la multiplicité explicative» (The Explanatory Multiplication Argument), une conclusion conditionnelle d'application qui limite les projections régies par les lois gouvernant les réalisateurs physiques $P$ de $M$.

Carl Gillet, dans un autre article "Non-Reductive Realization and Non-reductive Identity: What Physicalism Does Not Entail? ", soulève la question des propriétés émergentes. Il plaide, dans une véritable argumentation en faveur des propriétés fortement émergentes, pour un physicalisme non réductif à l'intérieur de ce qu'il nomme un physicalisme patchwork. Son objectif consiste ici à répondre à la solution de Kim au modèle classique de réduction proposé par E. Nagel. Pour Gillet, le modèle de réduction fonctionnelle préconisé par Kim ne doit pas, néanmoins, empêcher de considérer certaines propriétés comme véritablement irréductibles. Fondée sur le principe que les propriétés de niveau supérieur possèdent une véritable efficacité causale, la démonstration veut malgré tout rester en accord avec une des bases du physicalisme, à savoir que toutes les propriétés du niveau supérieur sont réalisées par des complexes de propriétés physiques fondamentales. Mais pour Gillet il existe aussi des lois de nature fondamentales qui impliquent, dans des lois de macro-niveau irréductibles, des propriétés fortement émergentes.

Enfin, et c'est le premier texte du recueil intitulé Multiply Realized Properties, J. Heil, discutant une position de la réalisation avancée par S. Shoemaker (Realization and Mental Causation) éclaircit nettement la différence qu'il faut établir entre propriétés et prédicats dans une perspective ontologique sérieuse. En un certain sens, le texte de Heil, bien que situé au début de cet ouvrage, pourrait se lire en conclusion de la discussion autour des travaux de Kim, tant cette explicitation semble éclairer le problème des propriétés réalisées de multiples façons.

Pour Heil, l'engagement du physicalisme non réductif en faveur de la réalisation multiple proviendrait de la tendance à considérer que les prédicats s'alignent parfaitement avec les propriétés. Ainsi, un grand nombre de philosophes estiment que les propriétés mentales sont réalisées de multiples façons, alors que l'ontologie des propriétés elles-mêmes resterait dans le flou.

L'argument général de Heil repose sur la notion de "sérieux ontologique». Cette notion est fondée sur un principe relatif aux vérifacteurs (Truth Maker): quand une affirmation prétendant à la vérité sur le monde est vraie, quelque chose du monde la rend vraie. Un tel principe s'oppose d'une certaine façon à l'idée que les prédicats seraient les ombres jetées par les prédicats. Heil, par un chemin inverse, nous propose donc de rendre leur dû aux propriétés.

Pour Heil, s'il existe des propriétés, elles appartiennent, à quelques exceptions près, à un type d'être non linguistique. Ainsi, parler de propriétés «disjonctives » ou "conjonctives » ou "conditionnelles ", ou encore de propriétés «entraînant " ceci ou cela n'a que très peu de sens.

Comme Kim, Heil abandonne les propriétés de niveau supérieur en faveur des prédicats de niveau supérieur. Mais, contrairement à Kim, il estime que la pertinence causale dans le fait de ressentir une douleur ou de croire que p n'est pas menacée, pour 
autant que le vérifacteur d'un prédicat comme «X ressent une douleur » soit une propriété physiquement réalisée, qui, elle, est sans controverse causalement pertinente.

FRANÇOIS LOTH

Université de Rennes

Jean-Marc Narbonne, «Lévinas et l'héritage grec», accompagné de Wayne Hankey, «Cent ans de néoplatonisme en France. Une brève histoire philosophique», trad. par M. Achard et J.-M. Narbonne, Paris et Québec, Vrin et Presses de l'Université Laval, coll. «Zêtêsis», 2004, 268 pages.

Ce livre regroupe deux études qui devaient être à l'origine deux articles. Comme ils s'en expliquent dans l'avant-propos, leurs auteurs ont souhaité en faire un volume, considérant qu'il y aurait là témoignage de la vigueur des études néoplatoniciennes en langue française et, avec l'exemple privilégié de Lévinas, une analyse de l'influence néoplatonicienne sur la phénoménologie française. J.-M Narbonne publie ainsi, dans une collection qu'il dirige, un essai sur le néoplatonisme apparent et implicite de Lévinas, et une co-traduction d'un essai d'«histoire philosophique» consacré aux principaux représentants français des études néoplatoniciennes ainsi qu'à l'influence de ces études sur la philosophie française contemporaine.

La thèse que J.-M. Narbonne entend défendre dans son essai est la suivante: la philosophie contemporaine, sous sa forme la plus inspirée, c'est-à-dire heideggérienne, n'a pas saisi l'importance de la méditation grecque d'un au-delà de l'être, de ce que J.-M. Narbonne (désormais JMN) appelle du nom presque propre d'epekeina ( «au-delà») ${ }^{1}$. Cette méditation, qui prend sa source dans Platon, trouve son accomplissement dans Plotin, et Heidegger s'est donc trompé en ne voyant dans la philosophie grecque qu'une réflexion sur l'étant. Certains de ses héritiers français, ou bien certains des historiens français de la philosophie dans les travaux desquels l'œuvre heideggérienne a produit des effets ou prononcé des injonctions, ont en revanche été plus attentifs, selon JMN, à l'importance d'une pensée de l'au-delà de l'être qu'on voit s'affirmer dans la théologie négative ancienne et dans la tradition platonicienne. Parmi eux, Lévinas figurerait donc en première place. C'est ce qu'entend montrer $\mathrm{JMN}$, qui procède de manière didactique en indiquant que ce qui a été manqué par Heidegger a été saisi par Lévinas et qui entreprend de repérer la manière dont Lévinas hérite d'une version "néoplatonisée » de l'epekeina platonicien. Une parenté est alors établie entre "l'au-delà de l'être» des néoplatoniciens et «l'autrement qu'être» du phénoménologue contemporain.

Afin de mieux établir sa démonstration, JMN dresse un décor ancien où la réflexion contemporaine trouvera son pendant: il soutient que la question de Dieu occupe la philosophie ancienne, qui se montrerait tout aussi soucieuse de l'epekeina. Ce faisant, JMN découvre sa dette à une tradition d'exégèse contemporaine dont on

1. Hénologie, ontologie et Ereignis. Plotin, Proclus, Heidegger, Paris, Belles Lettres, 2001. 